\title{
DÜBLIN
}

Technological University Dublin

ARROW@TU Dublin

\section{Refractive Index Sensor Based on a Silica Microsphere Whispering Gallery Mode Resonator}

\author{
Arun Mallik \\ Technological University Dublin \\ Qiang wu \\ Technological University Dublin, qiang.wu@tudublin.ie \\ Gerald Farrell \\ Technological University Dublin, gerald.farrell@tudublin.ie
}

See next page for additional authors

Follow this and additional works at: https://arrow.tudublin.ie/engschmanconn

Part of the Electrical and Computer Engineering Commons, and the Manufacturing Commons

\section{Recommended Citation}

Mallik, A. K, et al. (2015) Refractive Index Sensor Based on a Silica Microsphere Whispering Gallery Mode Resonator. Recent Advances in Photonics (WRAP), 2015 Workshop on. IEEE, 2015.doi:10.21427/e0wxt805

This Conference Paper is brought to you for free and open access by the School of Manufacturing and Design Engineering at ARROW@TU Dublin. It has been accepted for inclusion in Conference Papers by an authorized administrator of ARROW@TU Dublin. For more information, please contact arrow.admin@tudublin.ie, aisling.coyne@tudublin.ie,gerard.connolly@tudublin.ie.

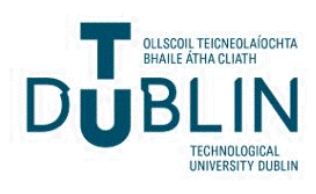




\section{Authors}

Arun Mallik, Qiang wu, Gerald Farrell, and Yuliya Semenova

This conference paper is available at ARROW@TU Dublin: https://arrow.tudublin.ie/engschmanconn/40 


\title{
Refractive Index Sensor Based on a Silica Microsphere Whispering Gallery Mode Resonator
}

\author{
Conference Paper · December 2015
}

DOI: 10.1109/WRAP.2015.7805968

CITATIONS

0

4 authors, including:

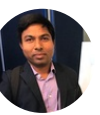

\section{Arun Mallik}

Dublin Institute of Technology

27 PUBLICATIONS 32 CITATIONS

SEE PROFILE

\section{Yuliya Semenova}

Dublin Institute of Technology

256 PUBLICATIONS 1,931 CITATIONS

SEE PROFILE
READS

176
Qiang Wu

Northumbria University

211 PUBLICATIONS 1,312 CITATIONS

SEE PROFILE

Some of the authors of this publication are also working on these related projects: 


\title{
Refractive Index Sensor Based on a Silica Microsphere Whispering Gallery Mode Resonator
}

\author{
Arun Kumar Mallik, Qiang Wu, Gerald Farrell, , Yuliya Semenova \\ Photonics Research Center \\ Dublin Institute of Technology \\ Dublin 8, Ireland \\ d13125763@mydit.ie
}

\begin{abstract}
We investigated the excitation of whispering gallery modes (WGMs) in a silica microsphere by evanescent field coupling method using a $\sim 2$ micrometer diameter fiber taper. Based on this configuration we have developed a sensitive refractometric sensor and observed a red-shift of the WGM resonances with the increase of the surrounding medium refractive index. A sensitivity of $\sim 114$ nanometer per RIU has been achieved in the range of refractive indices from 1.3963 to 1.4004
\end{abstract}

Keywords - Whispering Gallery Mode, Optical resonator, Optical fiber sensors, Refractive index

\section{INTRODUCTION}

Evanescent field based optical sensor is one of the most commonly used types of transducers for sensing applications in the fields of liquid, food and water-quality control, health, safety, and environmental monitoring. Among the evanescent field based sensors, whispering gallery mode (WGM) resonators have gained a substantial popularity in recent years due to their high quality factors $(\mathrm{Q})$, low absorption loss and easy and inexpensive fabrication methods. WGMs are high angular momentum modes which can be excited by trapping light propagating inside a dielectric structure with circular symmetry, such as a microsphere, by repeated total internal reflection. When light is reflected at the surface of the resonator, it induces the evanescent wave in the surrounding medium that propagates along the tangent to the surface and extends $\sim 200 \mathrm{~nm}$ outward [1]. The spectral responses of resonances are strongly dependent on the geometry of the dielectric resonator (diameter, sphericity), optical properties of the resonator material and also on the refractive index (RI) of surrounding the resonator medium, which makes this device suitable for sensing of the surrounding RI [2,3]. WGMs in a silica sphere can be easily excited by trapping a portion of evanescent field propagating through a fiber taper and the changes in the surrounding RI can be measured by monitoring either reflected or transmitted light through the taper [4].
In this paper we demonstrate application of WGMs in a silica microsphere for refractometric sensing. The WGMs are excited in the microsphere by evanescent light coupling using a $\sim 2 \mu \mathrm{m}$ tapered fiber. Without disturbing the microspheretaper alignment, a few drops of higher refractive index liquid were added. Resonance wavelength shift was recorded using photo detector with the help of labview programme and a sensitivity of $114 \mathrm{~nm} / \mathrm{RIU}$ was observed when the RI of the liquid changed from 1.3963 to 1.4004 in real time.

\section{THEORY}

Electromagnetic field distribution in a dielectric sphere depends on the sphere's effective radius, dielectric loss factor for different modes and the transmission coefficient between the coupled tapered fiber and the sphere. High resolution spectroscopy of whispering gallery modes in a large dielectric sphere $(\mathrm{R}>>\lambda)$ was reported by Stephan Schiller et al [5]. For large spheres the resonance frequency of WGM can be expressed as

$$
\begin{gathered}
\mathrm{n}_{\mathrm{s}} \mathrm{x}_{\mathrm{l}}{ }^{\mathrm{i}}= \\
v+\frac{\alpha_{\mathrm{i}}}{2^{1 / 3}} \mathrm{v}^{1 / 3}-\frac{\mathrm{mp}}{\sqrt{\mathrm{m}^{2}-1}}+\frac{3 \alpha_{\mathrm{i}}}{10 \times 2^{2 / 3}} v^{-1 / 3}+ \\
\frac{\mathrm{m}^{3} \mathrm{p}\left(\frac{2 \mathrm{p}^{2}}{3}-1\right) \alpha_{\mathrm{i}}}{2^{\frac{1}{3}}\left(\mathrm{~m}^{2}-1\right)^{3 / 2}} \mathrm{v}^{-2 / 3}+0\left(\mathrm{v}^{-1}\right)
\end{gathered}
$$

where $l$, is the angular mode number of the sphere, $v=l+$ $\frac{1}{2}, m=n_{s} / n_{e}$ is the relative RI between the sphere and the medium in which it is immersed, $p=1$ for TE modes, $p=1 / m^{2}$ for TM modes, $\alpha_{i}$ is the $i$ th zero of Airy function and $x=2 \pi R / \lambda$. The above equation states that RI change of immersion liquid results in a larger spectral shift for a lower angular mode. At the same time broadening in the line width of the mode also takes place. The resonance condition of the sphere is $N \lambda n_{s}=2 \pi R$, where $N$ is the integer number of the orbital wavelength. Based on this resonance condition the sensitivity of the resonance wavelength to changes in the 
radius $\Delta \mathrm{R}$ or refractive index $\Delta \mathrm{n}_{\mathrm{s}}$ is given by the following equation [6]:

$$
\frac{\Delta \lambda}{\lambda}=\frac{\Delta \mathrm{R}}{\mathrm{R}}+\frac{\Delta \mathrm{n}_{\mathrm{s}}}{\mathrm{n}_{\mathrm{s}}}
$$

It can be seen that an increase in the radius or the RI both lead to a red shift in the WGM resonance wavelength.

\section{EXPERIMENTAL SETUP}

The microsphere for our experiments was fabricated at the tip of a standard single mode fiber (Figure 1(a)). The cleaved end of the fiber was put inside a fusion splicer (Sumitomo Type-36). A series of electric arc discharges was applied to the cleaved part of the fiber, so that the tip of the fiber was gradually melted and became spherical in shape due to surface tension (Figure 1(b)). As reported previously in [7] the diameter of the sphere increases with the number of arcs reaching a saturation value around $350 \mu \mathrm{m}$. In this experiment spheres with a diameter circa $300 \mu \mathrm{m}$ were used. The sensitivity $(\mathrm{S}=\delta \lambda / \delta \mathrm{n})$ of the resonator to the surrounding RI increases with the decrease of the sphere diameter, however as was demonstrated in [3] the decrease in diameter also results in the reduction of the Q- factor. As Q factor implies how many round trips a photon can be travelled inside the sphere before being lost by absorption and scattering. In our experiments we used microsphere with diameters between 220 $\mu \mathrm{m}$ and $320 \mu \mathrm{m}$. The main advantage of such a size is ease of fabrication and handling during the experiment. Tapered fiber was fabricated using a customized micro heater brushing technique. A few centimeters of coating length was removed from the fiber before tapering. Then the fiber was placed horizontally using two computer controlled XYZ translational stages pulling the fiber through the micro heater opening. During tapering, translation stages were moved horizontally at the same time fiber was being stretched by the stages. A micrograph of the resulting taper is shown in Figure 1(c). After fabrication two ends of the taper were fixed with UV curable epoxy on the glass slide.

Optical characterization set up is shown in Fig (2). One end of the tapered fiber was connected to the Tunics-plus outside cavity tunable laser. This laser has a capability of wavelength tuning with a resolution of 1 pico meter within the range 1.3963 to 1.4054 . A LabView programme was developed to control the tuning of the laser wavelength. The laser output was passed through a motorized polarization controller. The microsphere and tapered fiber were mounted on a xyz nano-metric translation stage. Microsphere was slowly and carefully brought in contact with the tapered fiber for excitation of WGM resonances. The other end of tapered fiber was connected to a high speed InGaAs photo detector (Thorlabs PDA 10CS). The coupling between microsphere and tapered fiber was monitored by a high resolution digital camera.

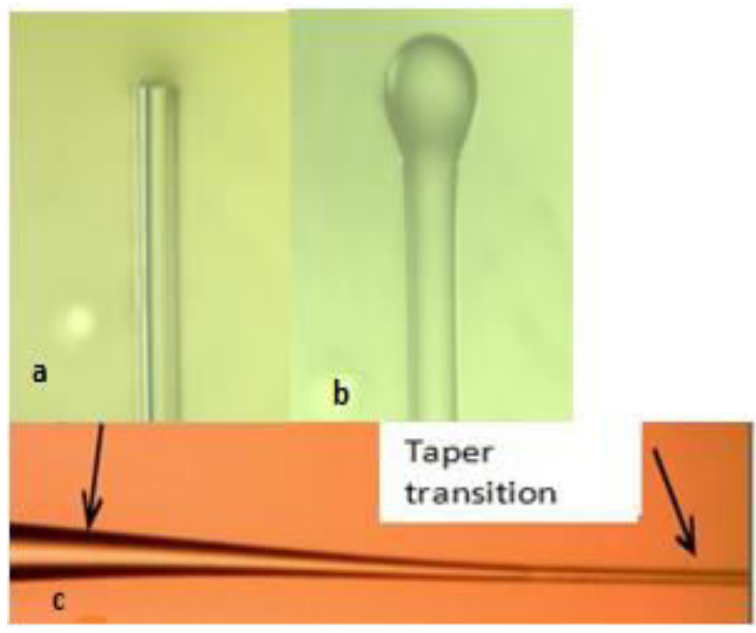

Fig .1. Optical fiber tip before (a) and after (b) applying an electric arc, (c) tapered fiber

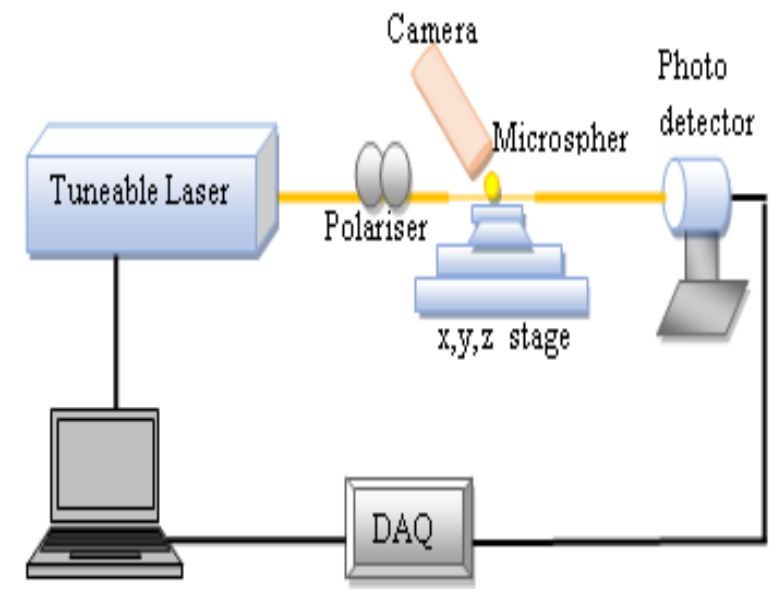

Fig. 2.Experimental setup for microsphere and fiber taper coupling

\section{RESULTS AND DISCUSSION}

Before conducting the refractive index sensing experiment, excitation of the WGM resonance was ensured passing the TE polarized laser light through the fiber taper which was evanescently coupled to the equatorial part of the microsphere. The $Q$ factor of the WGM resonance was estimated by measuring the line width of the WGM resonance around $1550 \mathrm{~nm}$ as shown in Figure 3. The fundamental WGM corresponds to the dips with the highest extinction ratio. The quality factor can be calculated as $Q=\frac{\lambda_{\text {res }}}{\Delta \lambda_{F W H M}}$, where $\lambda_{\text {res }}$ is the wavelength of a resonance, and $\Delta \lambda_{F W H M}$ is the width of the resonant lobe at full-width of half maximum (FWHM). The FWHM is calculated by fitting one of the best resonance dips to a Lorentz equation as shown in the inset of Figure 3.We estimated the FWHM as $0.085 \mathrm{pm}$ at the wavelength of $1550.5345 \mathrm{~nm}$. The corresponding value of the $Q$ - factor is calculated approximately as $1.8 \times 10^{7}$. 


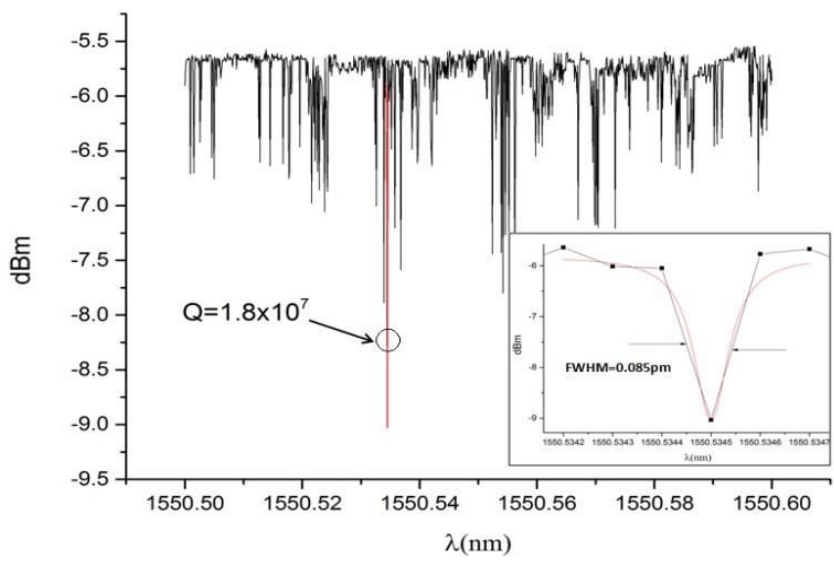

Fig 3: Experimental transmission spectrum for a $270 \mu \mathrm{m}$ diameter sphere coupled to a $\sim 2 \mu \mathrm{m}$ tapered fiber.

For the RI sensing experiment the liquid with refractive index 1.3963 was prepared by mixing of dimethyl sulphoxide with refractive index of 1.4790 and deionized water using a magnetic stirrer. Refractive index of the mixture was measured as 1.3963 by Abbey's refractometer. Few drops of the liquid mixture were carefully placed on the glass slide with the fiber taper. The glass slide was slowly moved upward toward the sphere until the sphere was immersed completely in the liquid without disturbing the coupling between sphere and tapered fiber. The transmission spectrum of immersed

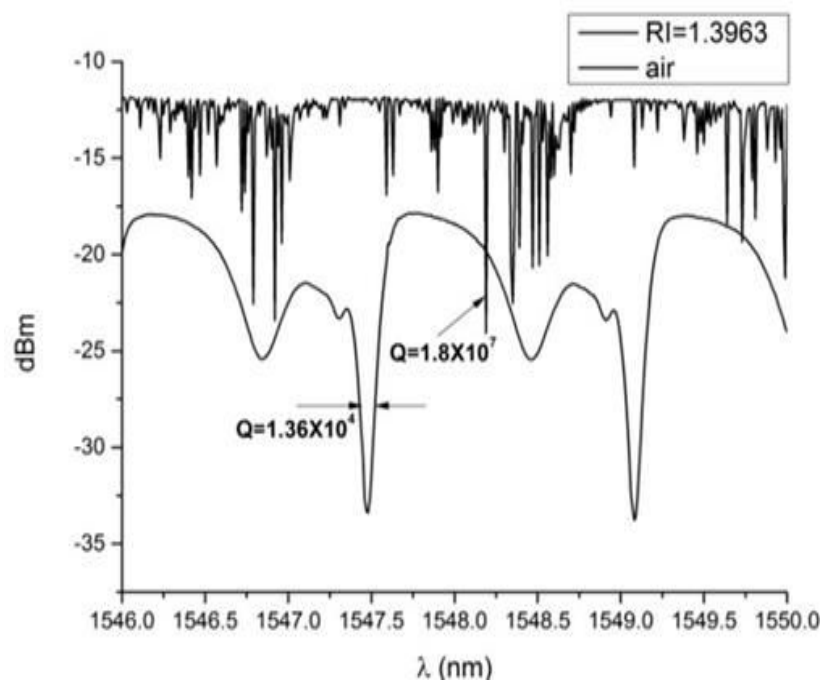

Fig: 4 Real time spectrum of the taper coupled to the microsphere immersed in a liquid of with refractive of 1.3963

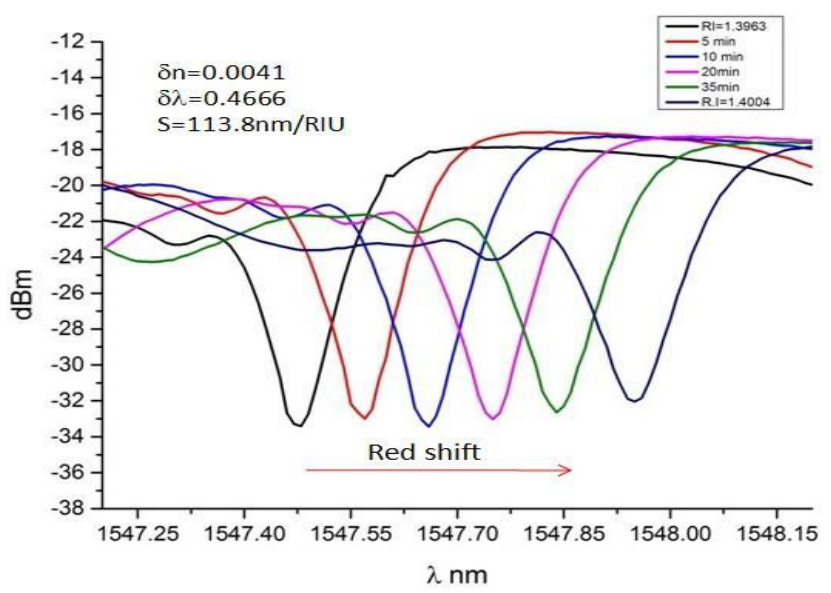

Fig 5: Red shift of $466 \mathrm{pm}$ of WGM spectrum when the refractive index of liquid increases $4 \times 10^{-3}$

sphere is shown in Figure 4.It can be clearly noticed from the figure that all other modes vanish after immersing the microsphere in the liquid due to the decrease in refractive index contrast in comparison with the case in air. The Q factor of the immersed in the liquid microsphere is significantly lower $\left(1.36 \times 10^{4}\right)$ compared to that in air $\left(1.8 \times 10^{7}\right)$ and is shown in Fig. 4.Tuning of the surrounding the microsphere refractive index was done by adding few drops of a higher refractive index liquid into the mixture. Simultaneously the WGM spectra were recorded after 5, 10, 20 and 35 min respectively. After 35 min we stopped recording the spectra as there was no further changes in the positions of the WGM resonances. An overall red shift of the WGM resonances 0.466 $\mathrm{nm}$ was observed when the refractive index of the liquid was changed from 1.3963 to 1.4004 .

\section{CONCLUSION}

In conclusion, we have reported the observation of refractive index sensing based on a spherical WGM resonator fabricated at the end tip of silica fiber Maximum demonstrated sensitivity of the WGM sensor to the surrounding refractive index change was $114 \mathrm{~nm} / \mathrm{RIU}$ in the range of RIs from 1.3963 to 1.4004 . This value could be significantly improved by fine tuning the parameters of the microsphere and thus such a sensor could be developed into a microprobe for label-free sensing for various applications.

\section{Acknowledgment}

Arun Kumar Mallik would like to acknowledge the support of Dublin Institute of Technology and Fiosraigh Dean of Graduate Students scholarship.

\section{References}

[1] F. Volmer and S. Arnold, "Whispering gallery mode biosensing: label free detection down to single molecules", Nature methods, vol. 5, pp. 591-596, June 2008. 
[2] T. Ioppolo, N. Das, and M. V. Otugen, "Whispering gallery modes of microsphires in the presence of a changing surrounding medium: A new ray-tracing analysis and sensor esperiment," J. Appl. Phys., Vol. 107, pp. 103105(1)-103105(8), Aug. 2010.

[3] J. C. Knight, G. Cheung, E Jacques, and T. A. Birks, "Phased-matched excitation of whispering-gallery mode resonances by a fiber taper," Opt. Lett. vol 25 p 1129-1131, 1997.

[4] N.M.Hanumegowda, C.J.Stica, B.C.Patel, I.White, and X.Fan, "Refractometric sensors based on microsphere resonators", Appl.Physc.Lett. 87 ,pp.201107-1-201107-3, Nov 2005.

[5] S. Schiller, R.L. Byer, "High-resolution spectroscopy of whispering gallery modes in large dielectric spheres", Opt. Lett., vol.15, pp.11381140 ,Aug 1991.

[6] F. Vollmer, D. Braun, and D. Keng, "Single virus detection from the refractive shift of a whispering gallery mode" Proc. Natl. Acd. Sci. U.S.A, vol.105, pp.20701-20704, Nov 2008.

[7] S. Pang, R.E. Beckham, K. E. Meissner, "Quantum dot-embedded microspheres for remote refractive index sensing", Appl.Phys.Lett.,vol.92,pp.221108, 2008. 